\title{
Efectividad de los abordajes terapéuticos aplicados en docentes con alteraciones vocales funcionales
}

\author{
Therapeutic Effectiveness of Approaches Applied \\ in Teachers with Functional Vocal Disorders
}

\section{Rodrigo Maximiliano Jerez \\ Fonoaudiólogo \\ Instituto de Comunicación Social, Imagen y Sonido, ICSIS}

\section{RESUMEN}

El presente trabajo constituye una revisión bibliográfica acerca de la efectividad de la terapia/entrenamiento funcional aplicada a docentes con alteraciones vocales funcionales. Se desarrolla un diseño de investigación observacional y retrospectivo, bajo la metodología de revisión sistemática, a partir de publicaciones de revistas en las bases de datos PubMed, SciELO, Scopus y Cochrane, así como en revistas especializadas como la de la American SpeechLanguage-Hearing Association (ASHA), Journal of Voice, CEFAC y Redalyc. La revisión del material evidencia resultados positivos para los distintos abordajes abarcados. En diversas proporciones, cada uno contribuye a mejorar la calidad vocal, sostener el equilibrio entre los componentes que construyen la emisión y prolongar la armonía que debe sustentar la voz.

Palabras clave: abordaje fisiológico, disfonías funcionales, docentes, terapia funcional.

\begin{abstract}
This paper constitutes a bibliographic review on the effectiveness of therapeutic/training approaches applied in teachers with vocal alterations. Research has been designed in a retrospective and observational way, with a systemic review methodology based on publications from papers indexed in PubMed, SciELO, Scopus and Cochrane databases, as well as specialized journals such as American Speech-Language-Hearing Association (ASHA), Journal of Voice, CEFAC and Redalyc. The review of the material showed positive results for the different approaches. In varying proportions each one contributes to improve voice quality, hold the balance between the components that build the emission and prolong the harmony which should sustain the voice.
\end{abstract}

Keywords: functional therapy, physiological approach, teachers, voice disorders.
Contacto con el autor: Rodrigo Maximiliano Jerez Buenos Aires - Argentina Correo-e: rodrigomjerez@yahoo.com.ar 


\section{Introducción}

La voz es el componente sonoro de la comunicación que sustenta la realización efectiva del lenguaje oral. En los individuos que utilizan la voz para el desempeño de un trabajo, como es el caso de los docentes, el uso incorrecto del instrumento vocal origina problemas en el bienestar comunicativo e interfiere en el desempeño correcto de la profesión. Las altas tasas de problemas de voz en los docentes surgen de las exigencias vocales en el trabajo (Ziegler, Gillespie \& Verdolini Abbott, 2010) y numerosos factores de riesgo, tales como tareas prolongadas sin los adecuados tiempos de reposo y hablar en entornos ruidosos o salones de clases muy amplios sin la acústica conveniente (Simberg, Sala, Tuomainen, Sellman \& Rönnemaa, 2006).

Por otro lado, la efectividad de los tratamientos en estos casos es determinante para quienes deben someterse a ellos, debido al tiempo destinado a rehabilitación y entrenamiento que brindan las Aseguradoras de Riesgo de Trabajo (o entidades equivalentes fuera de Argentina), sobrevalorándose por esta razón la eficacia y rapidez de dichas intervenciones. Cada una de estas acciones fueron aplicadas en un tiempo de entre 30 y 90 minutos una vez por semana, por un promedio de 12 sesiones (tres meses de trabajo) en poblaciones, en su mayoría, de docentes femeninas.

El objetivo de este trabajo fue analizar los resultados obtenidos en diversas investigaciones clínicas realizadas en poblaciones docentes y estudiantes de magisterio, afectadas por alteraciones o desórdenes vocales de tipo funcional, con quienes se trabajó desde un enfoque terapéutico fisiológico.
La metodología de revisión permitió analizar 15 publicaciones relacionadas con abordaje fisiológico o funcional en voz, que cumplieron con los criterios descritos en el objetivo. Según estas, se requería que los estudios fueran investigaciones clínicas realizadas en poblaciones docentes y estudiantes de magisterio, que presentaran alteraciones o desórdenes vocales de tipo funcional; y, en los cuales se hubiese aplicado una intervención terapéutica o de entrenamiento basado en un enfoque fisiológico.

\section{Terapia de enfoque funcional o fisiológico}

La característica principal de los métodos de intervención enfocados desde una óptica fisiológica es la no desarticulación, durante el desarrollo de los ejercicios propuestos, de los tres componentes básicos necesarios para la construcción del fenómeno vocal, a saber: función respiratoria, función fonatoria y función resonancial. Tres ejemplos de ello son Vocal Function Execises (VFE), técnica finlandesa con tubos de resonancia y terapia con voz resonante.

\section{Vocal Function Exercises (VFE)}

El programa terapéutico creado y articulado por J. Stemple (1994) fue usado en cinco de los 15 estudios considerados en la bibliografía primaria para el presente trabajo, y en tres casos se evalúa y compara con la terapia de Higiene Vocal (VH).

Roy et al. (2001) y Nanjudeswaran et al. (2012) revelan curiosamente resultados muy similares alrededor de estos métodos. Mientras el primero realizó estudios comparativos entre VH y VFE en una población de 58 docentes con desórdenes vocales funcionales divididos en tres grupos (VH $n=20$; VFE 
$n=18$ y grupo control $n=18$ ); el segundo ponderó $\mathrm{VH}$ y VFE + VH (Vocal Function Exercises junto con medidas de Higiene Vocal) en un grupo de 31 estudiantes de magisterio con valores altos y bajos en el Voice Handicap Index $(\mathrm{VHI})^{1}$. Ambos sugieren que el VFE sería considerado una alternativa útil conjunta con los programas de higiene vocal en el tratamiento para los problemas vocales en docentes y/o futuros docentes. Los beneficios percibidos por los pacientes del programa VFE fueron también verificados por cuestionarios posteriores al tratamiento completados por los participantes.

Cuando se compararon los grupos VH (Programa de Higiene Vocal) y VFE; el grupo que recibió ejercicios reportó mayores mejorías vocales, así como mayor claridad y facilidad en su voz después del tratamiento (Roy et al., 2001).

El programa de Higiene Vocal resultó suficiente solo para prevenir la decadencia en los puntajes del Voice Handicap Index (VHI), algo también observado en todos los participantes del grupo control. Para los sujetos con puntajes VHI inicialmente pobres, el programa de Higiene Vocal falló sin mostrar beneficios sobre la condición de control. La adición de terapia vocal fue requerida para optimizar los resultados en este grupo (Nanjudeswaran et al., 2012).

Por su parte, Alves de Mendoça, Marinho Sampaio y Ferreira de Oliveira (2010) aplicando VFE en un grupo de 17 profesoras reveló, luego de la terapia, un aumento del tiempo fonatorio, de la intensidad y de la calidad vocal, evidenciado a través

${ }^{1}$ El índice de incapacidad vocal o Voice Handicap Index (VHI) es un cuestionario desarrollado por Jacobson et al. (1997) con el fin de cuantificar el impacto percibido por un sujeto afectado por un trastorno vocal en los ámbitos de la propia función vocal, en la capacidad física relacionada con ella y en las emociones que provoca la disfonía (Nuñez Batalla et al., 2007). de una mayor presencia de armónicos en los espectrogramas analizados, además de ampliar la extensión de la voz y mejorar la calidad, resistencia y proyección vocal.

Este programa de ejercicios aportó mayores habilidades a los participantes para el desempeño profesional, beneficio extendido también al ámbito social. El programa VFE puede ser usado con eficacia, pues su efecto positivo se demostró en esta muestra de docentes.

En el trabajo de Niebudek-Bogusz y colaboradores (2008) se advirtió una mejora tanto objetiva como subjetiva (apreciación del propio paciente) en un grupo de 133 docentes femeninas con disfonía funcional de base, luego de la aplicación de VFE de dos a cuatro meses (entre nueve y 18 sesiones) con sesiones de 45 a 60 minutos. Los beneficios se dieron alrededor de diversos síntomas reportados, así como en algunos parámetros cuantitativos del examen foniátrico, comparado con los hallazgos en el grupo control $(n=53)$ formado por un grupo de docentes con idénticas características que no recibió indicación alguna. El número de pacientes que evaluaron sus voces como normal, incrementó significativamente después del entrenamiento vocal. De manera similar, se observó un aumento significativo en el tiempo máximo fonatorio (TMF).

Por su parte, Gillivan-Murphy, Drinnan, O'Dwyer, Ridha y Carding (2006) examinaron la eficacia de un tratamiento combinado entre los enfoques VFE y VH, en un estudio a medio camino entre Roy (2001) y Nanjudeswaran et al. (2012), sugiriendo a través de una investigación prospectiva realizada durante seis semanas en 20 docentes con problemas vocales, que 
un tratamiento vocal de VFE y educación VH mejoran los síntomas vocales y aportan positivamente a los conocimientos mínimos para el cuidado de la voz del grupo de estudio, lo que se traduce en una mayor conservación vocal.

\section{Tubos de resonancia}

Esta estrategia, que lleva vigente más de 40 años, sigue ofreciendo resultados positivos y alentadores en sus estudios. Sobre el total de estudios primarios, tres de ellos llevaron a ensayo este método.

En Simberg et al. (2006), las pruebas se desarrollaron sobre la base de 20 estudiantes de magisterio, poniendo a prueba la eficacia de esta modalidad de abordaje. Dos de los tres resultados considerados aquí (evaluación perceptual de la calidad vocal realizada por profesionales entrenados y cuestionario acerca de los síntomas vocales respondido de manera individual por los participantes del estudio) indicaron cambios significativos entre el grupo que recibió tratamiento y aquel que no fue tratado (grupo control formado por 20 sujetos con iguales características vocales). Por otra parte, no hubo diferencias notorias en el estado laríngeo entre ambos conjuntos. Los resultados sugieren que este enfoque es un método efectivo para tratar estudiantes con alteraciones vocales funcionales.

Frente a los restantes estudios clínicos analizados, cabe aclarar que los efectos inmediatos de los ejercicios con tracto vocal semiocluido ya han sido descritos por la literatura contemporánea, sin embargo, no se había explicado lo referente a la técnica finlandesa con tubos de resonancia.

En esta línea, Guzmán, Higueras, Fincheira,
Muñoz y Guajardo (2012) concluyen en un estudio realizado sobre la base de 24 profesores con voces disfónicas, que los cambios significativos durante su experiencia fueron obtenidos en el análisis Cepstral, Jitter y relación armónico-ruido. En la autovaloración, los efectos positivos predominaron sobre los negativos, siendo los principales "voz más estable", "voz más clara" y "garganta más abierta". De esta manera, concluyen que el uso de tareas fonatorias dentro de tubos de resonancia tiene un efecto fisiológico terapéutico inmediato en sujetos docentes con voces disfónicas, perceptualmente clasificadas como sopladas.

El cambio de parámetros acústicos, la percepción subjetiva de mejoría y facilidad en la producción de la voz obtenida posteriormente a la terapia se explicaría por el cambio de patrón vibratorio de los pliegues vocales causado por la mayor interacción fuente-filtro al hacer uso de tubos de resonancia, según se expone.

Mazzer Paes, Zambon, Yamasaki, Simberg y Behlau (2013), sugieren efectos positivos inmediatos asociados con ejercicios con tubos de resonancia. Los 25 sujetos femeninos involucrados (con disfonía de hasta cinco años de data), por su parte, manifestaron una voz más confortable, menos tensa y exigida. El análisis auditivo perceptual mostró efectos positivos de los ejercicios en el número de muestras contemplado. El análisis espectrográfico de las vocales sostenidas por medio del programa VoxMetria ${ }^{2}$, reveló cambios favorables en casi todos los parámetros. Se redujeron la inestabilidad, los subarmónicos y el ruido en frecuencias altas, y una

${ }^{2}$ Software específico para análisis de voz y calidad vocal, con una gran variedad de funciones y parámetros, permitiendo al foniatra realizar el acompañamiento y comparaciones entre archivos de un mismo cliente y entre modelos de voz. 
tendencia hacia la reducción del ruido en frecuencias bajas.

El método de tubos de resonancia finlandeses produce cambios vocales de una fonación hiperfuncional a un mayor estado fonatorio de balance. Los sujetos, al concluir este estudio, identificaron efectos positivos tales como una mayor calidad vocal y confort fonatorio.

\section{Terapia con voz resonante}

Este enfoque terapéutico se basa en dos principios básicos: la obtención de sensaciones vibratorias a nivel anterior y la sensación de producción de voz fácil y fue escrutado por Chen y colaboradores (2007).

Ellos observaron, en un grupo de 24 docentes femeninas con disfonía funcional, que luego de aplicar la terapia durante 90 minutos, una sesión semanal durante ocho semanas, la severidad de la ronquera, tensión, monotonía, resonancia, ataque duro, y frito glótico en la percepción auditiva disminuyeron; también la acritud de la patología de cuerdas vocales, onda mucosa, amplitud, y cierre glótico en examinación videoestroboscópica, la presión umbral y los valores en la escala física del VHI fueron significativamente reducidos.

En consecuencia, la frecuencia fundamental de habla, el rango máximo de frecuencia fundamental de habla y el rango máximo de intensidad de habla fueron reducidos también después de la terapia. Por el contrario, no se encontraron cambios significativos en perturbación y medidas respiratorias posterapia, lo cual permite afirmar su efectividad para alteraciones vocales en el grupo docente referido.

\section{Discusión}

Los docentes son la población de uso vocal con mayor porcentaje y tendencia a presentar trastornos fonotraumáticos en relación con cualquier otra profesión (Roy et al., 2004; Russell, Oates \& Greenwood, 1998; Sapir, Keidar, \& Mathers-Smith, 1993; Smith, Gray, Dove, Kirchner \& Heras, 1997). Ahora bien, pese a que no ha sido la finalidad de este estudio analizar los riesgos ni las condiciones que predisponen al desarrollo de alteraciones vocales, cada uno de los trabajos consultados los ha considerado al momento de diseñar sus protocolos de prueba.

Sin embargo, después de examinados no puntualizan minuciosamente ni con claridad el nivel educativo en el cual los docentes ejecutan su labor ni la tarea específica que desempeñan relacionada con una posible especialidad (lo que se conoce en otros contextos como docentes de materias especiales, es decir, educación física, música, docente a cargo del grado). Se sabe que los docentes de preescolar requieren mayor exigencia vocal que los de primaria y/o secundaria o incluso que los universitarios. Sería interesante ampliar los estudios hacia estas dimensiones, desde el mismo enfoque ofrecido aquí.

Así mismo, estos estudios clínicos son referidos casi exclusivamente a la población femenina. Se entiende, pues, que en la mayoría de los países es más común que sea el género mencionado quien realice la labor docente. Desde esta perspectiva, sería preciso atender a este aspecto con particular atención en posteriores estudios, ya sean estos comparativos o descriptivos, para explorar la relación entre mejoría vocal, terapia/entrenamiento vocal, tipo de abordaje y su relación con el género o 
especialidad docente, haciendo énfasis en una población estrictamente masculina y/o mixta.

La totalidad de los estudios expuestos demuestra efectividad para dicho enfoque, donde se aplicaron terapias con un promedio de duración de 30 a 90 minutos una vez por semana por alrededor de 12 sesiones (tres meses de trabajo), que es el tiempo necesario para empezar a notar cambios vocales estables que beneficien la calidad sonora y laboral. No obstante, la población considerada ha tenido como característica basal una disfonía funcional leve, es decir, no hay mayores estudios que indiquen el grado de mejoría en relación con el tiempo y tipo de patología de corte orgánica (por ejemplo, patología nodular de larga data o quistes).

\section{Conclusión}

Los estudios consultados para el presente trabajo consideran la geografía de las alteraciones funcionales y de los desequilibrios vocales. Al ser realizados en diversas partes del mundo permiten concluir que se trata una problemática que se enmarca en el ámbito mundial.

Los resultados y conclusiones de los estudios arrojan una inclinación evidente hacia un progreso vocal en los pacientes posterapia según el enfoque funcional.

La serie de Ejercicios Funcionales Vocales (VFE), en algunos casos como método exclusivo y otros como parte de un programa más extenso, considera estos ejercicios como una útil alternativa de rehabilitación/entrenamiento vocal para docentes con problemas de voz.

Los estudios clínicos basados en ejercicios con tubos de resonancia evidenciaron una mejora inmediata en la percepción subjetiva de la voz y en su calidad. En esta línea, Ziegler et al. (2010) analizaron en varios estudios el impacto de ambas estrategias, y concluyeron de igual modo que los VFE y ejercicios con tubos de resonancia producen resultados positivos en docentes con alteraciones funcionales a nivel vocal.

El examen realizado en terapia con voz resonante también asegura su efectividad para alteraciones vocales en docentes.

Es de público conocimiento que la promoción y la prevención de la salud vocal y un correcto entrenamiento son la base formativa para que la población a la que se ha hecho referencia a lo largo de estas líneas, se vea cada vez menos afectada por problemas vocales; sin embargo, también se acepta que esas consideraciones aún no han llegado al grado de políticas de primera categoría como para que los diversos sistemas que sostienen la educación (instituciones gubernamentales, educativas, de salud y/o gremiales) las implementen como medidas imprescindibles y adecuadas.

Por otro lado, al verificar la efectividad de las estrategias de abordaje funcional puestas en consideración, es posible asegurar que con este tipo de enfoque y acercamiento terapéutico no solo se consigue salvaguardar, sino también restaurar de forma eficiente y eficaz la salud vocal en los docentes. 


\section{Referencias}

Batalha Ribeiro, M., Côrtes Gama, A. C., Barreto Bassi, I., \& Caldas Teixeira, L. (2013). Vocal, laryngeal and selfperception measurements of dysphonic teachers: Analysis after voice therapy. Revista CEFAC, 15(3), 631-640. http://dx.doi.org/10.1590/s1516-18462013000300016

Hwa Chen, S., Hsiao, T-Y., Hsiao, L-C., Chung, Y-M., \& Chiang, S-C. (2007). Outcome of resonant voice therapy for female teachers with voice disorders: Perceptual, physiological, acoustic, aerodynamic and functional measurements. Journal of Voice, 21(4), 415-425. http://dx.doi.org/10.1016/j.jvoice.2006.02.001

Gillivan-Murphy, P., Drinnan, M. J., O'Dwyer, T. P., Ridha, H. \& Carding, P. (2005). The effectiveness of a voice treatment approach for teachers with self-reported voice problems. Journal of Voice, 20(3), 423-431. http://dx.doi.org/10.1016/j.jvoice.2005.08.002

Guzmán, M., Higueras, D., Fincheira, C., Muñoz, D., y Guajardo, C. (2012). Efectos acústicos inmediatos de una secuencia de ejercicios vocales con tubos de resonancia. Revista CEFAC, 14(3), 471-480. http://dx.doi.org/10.1590/s1516-18462011005000127

Leppänen, K., Laukkanen, A. M., Ilomäki, I., \& Vilkman, E. (2009). A comparison of the effects of Voice Massage ${ }^{T M}$ and voice hygiene lecture on self-reported vocal well-being and acoustic perceptual speech parameters in female teachers. Folia Phoniatrica et Logopaedica, 61(4), 227-238. http://dx.doi.org/10.1159/000228000

Mazzer Paes, S., Zambon, F., Yamasaki, R., Simberg, S., \& Behlau, M. (2013). Immediate effects of the Finnish Resonance Tube Method on behavioral dysphonia. Journal of Voice, 27(6), 717-722. http://dx.doi.org/10.1016/j.jvoice.2013.04.007

Mendonça Alves de, R., Marinho Sampaio, T. M., \& Ferreira de Oliveira, D. S. (2010). Avaliação do programa de exercícios funcionais vocais de Stemple e Gerdeman em professores. Revista CEFAC, 12(3), 471-482. http://dx.doi.org/10.1590/s1516-18462010005000069
Nanjundeswaran, C., Y. K. Li, N., M. K. Chan, K., Richard, K. S., W., M.-L Yiu, E., \& Verdolini-Abott, K. (2012). Preliminary data on prevention and treatment of voice problems in student teachers. Journal of Voice, 26(6), 816.e1-816.e12.

http://dx.doi.org/10.1016/i.jvoice.2012.04.008

Niebudek-Bogusz, E., Sznurowska-Przygocka, B., Fiszer, M., Kotyło, P., Sinkiewicz, A., Modrzewska, M., \& SliwinskaKowalska, M. (2008). The effectiveness of voice therapy for teachers with dysphonia. Folia Phoniatrica et Logopaedica, 60(3), 134-141. http://dx.doi.org/10.1159/000120290

Núñez-Batalla, F., Corte-Santos, P., Señaris-González, B., Llorente-Pendás, J. L., Górriz-Gil, C., \& Suárez Nieto, C. (2007). Adaptación y validación del índice de incapacidad vocal (VHI-30) y su versión abreviada (VHI-10) al español. Acta Otorrinolaringológica Española, 58(9), 386-392. http://dx.doi.org/10.1016/s0001-6519(07)74954-3

Pasa, G., Oates, J., \& Dacakis, G. (2007). The relative effectiveness of vocal hygiene training and vocal function exercises in preventing voice disorders in primary school teachers. Logopedics Phoniatrics Vocology, 32(3), 128-140. http://dx.doi.org/10.1080/14015430701207774

Roy, N., Gray, D. S., Simon, M., Dove, H., Corbin-Lewis, K., \& Stemple, J. C. (2001). An evaluation of the effects of two treatment approaches for teachers with voice disorders: A prospective randomized clinical trial. Journal of Speech, Language, and Hearing Research, 44(2), 286-296. http://dx.doi.org/10.1044/1092-4388(2001/023)

Roy, N., Weinrich, B., Gray, S. D., Tanner, K., Stemple, J. C., \& Sapienza, C. M. (2003). Three treatments for teachers with voice disorders: A randomized clinical trial. Journal of Speech Language and Hearing Research, 46(3), 670-688. http://dx.doi.org/10.1044/1092-4388(2003/053)

Roy, N., Merrill, R. M., Thibeault, S., Parsa, R. A., Gray, S. D., \& Smith, E. M. (2004). Prevalence of voice disorders in teachers and the general population. Journal of Speech Language and Hearing Research, 47(2), 281-293. http://dx.doi.org/10.1044/1092-4388(2004/023) 
Russell, A., Oates, J., \& Greenwood, K. M. (1998). Prevalence of voice problems in teachers. Journal of Voice, 12(4), 467-497. $\quad$ http://dx.doi.org/10.1016/s08921997(98)80056-8

Sapir, S., Keidar, A., \& Mathers-Smith, B. (1993). Vocal attrition in teachers: Survey findings. International Journal of Language \& Communication Disorders, 28(2), 177-185. http://dx.doi.org/10.3109/13682829309041465

Simberg, S., Sala, E., Tuomainen, J., Sellman, J., Rönnemaa, A. M. (2006). The effectiveness of group therapy for students with mild voice disorders: A controlled clinical trial. Journal of Voice, 20(1), 97-109. http://dx.doi.org/10.1016/j.jvoice.2005.01.002

Smith, E., Gray, S. D., Dove, H., Kirchner, L., \& Heras, H. (1997). Frequency and effects of teachers voice problems. Journal of Voice, 11(1), 81-87. http://dx.doi.org/10.1016/s0892-1997(97)80027-6

Stemple, J. C., Lee, L., D’Amico, B., \& Pickup, B. (1994). Efficacy of vocal function exercises as a method of improving voice production. Journal of Voice, 8(3), 271278. http://dx.doi.org/10.1016/s0892-1997(05)80299-1

Ziegler, A., Gillespie, A. I., \& Verdolini Abbott, K. (2010). Behavioral treatment of voice disorders in teachers. Folia Phoniatrica et Logopaedica, 62(1-2), 9-23. http://dx.doi.org/10.1159/000239059 